\title{
TRI-SERVICE SURGICAL MEETING
}

The Tri-Service Surgical Meeting took place at Keogh Barracks, Ash Vale on 11-12 March 2005. There were excellent presentations in both the higher Surgical Trainee (HST) and Basic Surgical Trainee (BST) Prize sessions. The key note speech was given by Mr David Lane on surgery in West Africa for Medecin Sans Frontieres.

The joint winners of the HST prize were Maj JP Garner RAMC and Surg Lt Cdr C Parry RN presenting related research on resuscitation of blast and haemorrhage. The BST prize winner was Surg Lt Cdr S Barton RN who presented a fascinating case of hand reconstruction after significant injury in Iraq. Both prizes were kindly provided by the Military Surgical Society.

\section{HST PRIZE SESSION}

Development Of A Large

Animal Model For Investigating Resuscitation After Blast And Haemorrhage

J Garner, C Parry, J Bird, S Watts, E Kirkman

\section{Introduction}

Blast injury is an increasing threat on the modern battlefield from the proliferation of enhanced blast weapons. Some of those injured are also likely to suffer haemorrhage. BATLS now advocates hypotensive resuscitation for the hypovolaemic battlefield casualty but no research has investigated whether this strategy is appropriate after blast and haemorrhage. Our aim was to develop a large animal model of blast and haemorrhage with sufficient post-insult survival time to evaluate the response to resuscitation strategies.

\section{Methods}

Large White pigs were prepared surgically for cardiovascular monitoring. Animals received either blast or sham blast followed by a controlled haemorrhage of $25-40 \%$ total blood volume (BV), a shock phase and uncontrolled haemorrhage from a standardised aortotomy at the onset of resuscitation.

\section{Results}

Pilot studies indicated that a $40 \%$ haemorrhage and uncontrolled blood loss were too severe after blast. The uncontrolled element was omitted and the controlled blood loss reduced to $30 \% \mathrm{BV}$. This gave a severe hypotension that was augmented by blast.

The animals survived a 5 min shock phase. With aggressive fluid resuscitation all animals subjected to haemorrhage after sham blast survived for $8 \mathrm{~h}$ while of those given haemorrhage after blast $100 \%$ survived for $3.5 \mathrm{~h}$ and $67 \%$ survived to the study endpoint of $8 \mathrm{~h}$.

\section{Conclusion}

We have developed a model of blast and haemorrhage suitable to study the physiological response to resuscitation. Blast significantly modifies the response to blood loss in this model.

\section{Comparison OfThe Effects \\ Of Prolonged Resuscitation Strategies On Survival, After Combined Blast And Haemorrhagic Injuries}

\section{Parry, J Garner, J Bird, S Watts, E Kirkman}

\section{Introduction}

Uncontrollable haemorrhage is a leading cause of death in combat and accounts for $49 \%$ of deaths on the battlefield (prehospital). The escalating use of IED's and potentially thermobaric weapons has increased the likelihood of coalition casualties sustaining combined exsanguinating and blast injuries. The aim of this study was to compare the effectiveness of normotensive

\begin{tabular}{|l|l|l|l|l|}
\hline & \multicolumn{2}{|l|}{ Sham Blast $(\mathrm{n}=6)$} & \multicolumn{2}{l|}{ Blast (n=6) } \\
\hline & $\begin{array}{l}\text { Pre } \\
\text { haemorrhage }\end{array}$ & $30 \% \mathrm{BV}$ loss & $\begin{array}{l}\text { Pre } \\
\text { haemorrhage }\end{array}$ & $30 \% \mathrm{BV}$ loss \\
\hline MBP (mmHg) & $110 \pm 11$ & $45 \pm 4$ & $122 \pm 8$ & $26 \pm 4^{\star}$ \\
\hline HR (beats/min) & $155 \pm 29$ & $211 \pm 12$ & $135 \pm 26$ & $178 \pm 8^{\star}$ \\
\hline
\end{tabular}

MBP, Mean Arterial Blood Pressure; HR, Heart Rate; ${ }^{\star} P<0.05$ (ANOVA) vs corresponding sham blast value. Values are mean $\pm S E M$ 


\begin{tabular}{|l|l|l|l|}
\hline \multicolumn{2}{|c}{} & \multicolumn{1}{l}{$\begin{array}{l}\text { No. Surviving to } \\
8 \text { Hrs / Total }\end{array}$} & $\begin{array}{l}\text { Mean Survival time } \\
(95 \% \text { CI }) \text { min }\end{array}$ \\
\hline \multirow{2}{*}{ ATLS } & SHAM & $8 / 8$ & 480 \\
\cline { 2 - 4 } & BLAST & $4 / 6$ & $422(313-531)$ \\
\hline \multirow{2}{*}{ BATLS } & SHAM & $5 / 8$ & $352(210-494)$ \\
\cline { 2 - 4 } & BLAST & $0 / 6$ & $137(94-181)$ \\
\hline \multirow{2}{*}{ HSD } & SHAM & $4 / 6$ & $356(154-557)$ \\
\cline { 2 - 4 } & BLAST & $0 / 6$ & $97(17-176)$ \\
\hline
\end{tabular}

(ATLS) and hypotensive (BATLS) resuscitation after blast and haemorrhage. Further we compared isotonic saline with hypertonic saline dextran.

\section{Methods}

Large White pigs were surgically prepared under terminal anaesthesia. The animals were randomised to receive a standardised blast injury or sham. Subsequently all animals underwent a controlled haemorrhage of $30 \%$ of their estimated total blood volume. Animals were further randomised to receive one the following resuscitation strategies:

ATLS $0.9 \%$ saline, target systolic blood pressure SBP $\geq 110 \mathrm{mmHg}$; BATLS $0.9 \%$ saline, target SBP $\geq 80 \mathrm{mmHg}$; HSD $7.5 \%$ saline and $6 \%$ dextran target $\mathrm{SBP} \geq 80$ $\mathrm{mmHg}$

The primary end point was survival to 8 hours. All surviving animals were killed humanely.

\section{Results}

Hypotensive resuscitation with either $0.9 \%$ saline (BATLS) or with HSD significantly reduced survival time compared to normotensive (ATLS) resuscitation $(\mathrm{P}<0.0001$, Peto log rank). Further analysis revealed that the shortened survival time associated with hypotensive resuscitation achieved statistical significance in those animals subjected to blast $(\mathrm{P}=0.0003)$ but not in those given sham blast $(\mathrm{P}=0.066)$. There was no significant difference in survival time between those resuscitated hypotensively with $0.9 \%$ saline and HSD $(\mathrm{P}=0.718)$.

\section{Conclusion}

Prolonged hypotensive resuscitation strategies are not compatible with survival after blast and haemorrhagic injuries. Although hypertonic resuscitation fluids have been shown to have benefit in other circumstances they do not improve survival in this model of blast and haemorrhage.

\section{The Use Of Flammacerium In British Burns Units}

\section{J Garner, PSJ Heppell}

\section{Introduction}

Flammacerium (cerium nitrate-silver sulphadiazine) is only available in UK on a named patient basis. Anecdotally it is widely used in civilian burns units although little literature exists regarding its use in Great Britain. We designed a postal survey to assess the use of Flammacerium countrywide and whether its 'named patient only' designation causes problems in treating burns patients.

\section{Methods}

Postal questionnaires were sent to the directors of the 18 adult burns units in Great Britain and Ireland requesting information on the use of Flammacerium. The indications and limitations of its use were recorded, as were any problems obtaining supplies. Finally, burns units were asked if they would like to see Flammacerium fully licensed in this country.

\section{Results}

Replies were received from 14 units (response rate $=78 \%$ ). Six use Flammacerium frequently, six occasionally and two units never use it. It was generally used in the belief that it reduces the inflammatory response to burn injury (8/12), decreases bacterial colonization $(10 / 12)$ and provides a firm eschar for easier wound management (11/12). Although 8 units had no problems with supplies of the agent, 10 respondents indicated they would like to see Flammacerium fully licensed in UK.

\section{Conclusions}

Flammacerium is a widely used topical agent in the United Kingdom for deep dermal and full thickness burns in patients not undergoing early complete excision. It is used in the belief that it reduces bacterial colonisation of, and inflammatory response to, the burn wound and provides a firm, easily manageable eschar. Most burns units would like to see Flammacerium fully licensed in UK.

*Previously presented by Maj Heppell to TriService Plastic Surgery Meeting 2004. 


\section{Wastage Of Allogeneic Blood \\ Can Be Reduced By \\ Organisational Change: \\ A Local Experience}

\author{
GC Evans, V Clark, C Howie, \\ L Manson
}

\begin{abstract}
Aims
Allogeneic blood is a limited resource and should be managed appropriately to minimize wastage. Blood returned to Blood Banks contribute to avoidable wastage; around 20\% cannot be reissued, and are destroyed. The Crossmatch: Transfusion ratio measures efficiency of blood use and should be < 2 (SIGN Guideline No 54). C:T ratios in three patient groups were reviewed to assess the impact of organisational change within Elective Orthopaedics and the Blood Transfusion Service.
\end{abstract}

\section{Methods}

A retrospective audit reviewed data from three patient groups undergoing knee or hip arthroplasty (primary \& revision). Group one underwent surgery at an elective orthopaedic hospital (off-site Blood Bank), group two at the Royal Infirmary of Edinburgh (RIE: on-site Blood Bank) before the introduction of electronic blood issue, and group three at RIE after the introduction of electronic issue.

\section{Results}

\begin{tabular}{|l|l|l|l|l|l|}
\hline Group & Date & Patients & $\begin{array}{l}\text { Units } \\
\text { Crossmatched }\end{array}$ & $\begin{array}{l}\text { Units } \\
\text { Transfused }\end{array}$ & C:T Ratio \\
\hline 1 & Jun 00 & 96 & 224 & 80 & 2.8 \\
\hline 2 & Jun 02 & 137 & 98 & 58 & 1.56 \\
\hline 3 & Jun 04 & 135 & 147 & 122 & 1.18 \\
\hline
\end{tabular}

\section{Conclusions}

Historically, many allogeneic blood users have ordered blood on a 'just in case' basis to ensure timely blood availability; this results in return of unused blood.

With an appropriately equipped on site Blood Bank, blood can be supplied by electronic issue. This system facilitates the issue of group-specific blood within five minutes of the request being made. Blood can be ordered on a 'need to transfuse' basis and issued unit by unit immediately prior to transfusion, resulting in a low return rate, a C:T ratio approximating 1 and a significant reduction in wastage.

\section{Screening And Isolation To Control MRSA Infection In Vascular Surgical Patients}

\author{
ML Davies, O Ehsan, C Davies, \\ A Shandall, D McLain
}

\section{Introduction}

Infection of prosthetic vascular grafts is associated with a high mortality and morbidity. Prophylactic antibiotics have not been found to be very effective in the prevention of MRSA. We have completed a study to investigate the effects of screening and isolation on incidence of vascular graft infection.

\section{Materials and Methods}

An audit was carried out to determine the graft infection rate in our vascular surgery unit. This was followed by introduction of strict infection control measures, including screening for MRSA and isolation of vascular patients requiring bypass graft surgery. The outcomes following the introduction of screening and isolation procedures were investigated and the results compared.

\section{Results}

There was a reduction in graft infection rate of from $3.4 \%$ to $1.09 \%$. It was noted that most of the infections occurred when the vascular ward was not isolated and hence the actual reduction was to $0.36 \%(P=0.0066)$. The reduction in aortic graft infection rate was from $1.62 \%$ to $0 \%$.

\section{Conclusion}

Isolation of clean vascular patients in a separate ward, screening of patients prior to admission and strict infection control measures can decrease the graft infection rate. Lack of isolation on the vascular ward reverses the situation.

\section{Inhibition Of Angiogenesis By Prostasomes}

\section{G Delves, A Goyal, A Cooper}

\section{Introduction}

Prostasomes are biologically active organelles that are secreted by human prostate epithelial cells in both health and disease. It is believed that they have a role in prostatic disease, particularly adenocarcinoma. We studied the effect of prostasomes on angiogenesis, a key feature of tumour development, using the rat aortic ring model.

\section{Method}

Prostasomes were prepared from pooled post-vasectomy semen samples. Rat aortic 
rings were harvested from 8-week old male rats, placed in a Matrigel and medium matrix, and after $24 \mathrm{~g}$ hours treated with either test agent or control. Angiogenesis was quantified after 5 days by spectrophotometric assessment of cell viability. The effect of boiled prostasomes and artificially prepared liposomes was also assayed.

\section{Results}

Prostasomes were demonstrated to significantly inhibit angiogenesis activity, with $25.4 \%$ less absorption on spectrophotometric assay. This figure under represents the reduction of neovessel formation, as the ring itself contains many cells. Significant, though lesser, inhibition was also seen with preparations of boiled prostasomes and liposomes.

\section{Conclusion}

This study shows the in vitro inhibition of angiogenesis by prostasomes, contrary to previous speculation. The effectiveness of boiled prostasome preparations, in which protein has been denatured, suggests that this inhibition is not protein dependant. This is similar to other known prostasomal actions, in which transfer of lipids has been demonstrated and shown to be responsible.

\section{BST PRIZE SESSION}

\section{Healing Hands: Management Of A Shrapnel Wound Injury In The Dominant Hand Of A Serviceman, Sustained In The Iraq Conflict}

\section{SJ Barton, S Kaleel, S Rayatt, F Peart}

We report the case of a 35 year old serviceman who sustained multiple injuries to the eye, chest shoulder and right hand after his armoured vehicle was struck by multiple rocket propelled grenades. Managed initially in a Field Hospital by ATLS protocols and then surgical exploration in theatre: the right shoulder wound extended into the upper mediastinum and after median sternotomy was found to have lacerated the left innominate vein which was ligated. His right hand injury was a high energy transfer IIIa wound, heavily contaminated with metal fragments and powder burns. There was minimal skin loss and an open comminuted fracture of the index metacarpal - only limited debridement was performed because of his other overwhelming injuries.

He was transferred to our hospital on Day 3, whereupon his chest wounds were reexplored and his ocular injury managed surgically. When stable his grossly

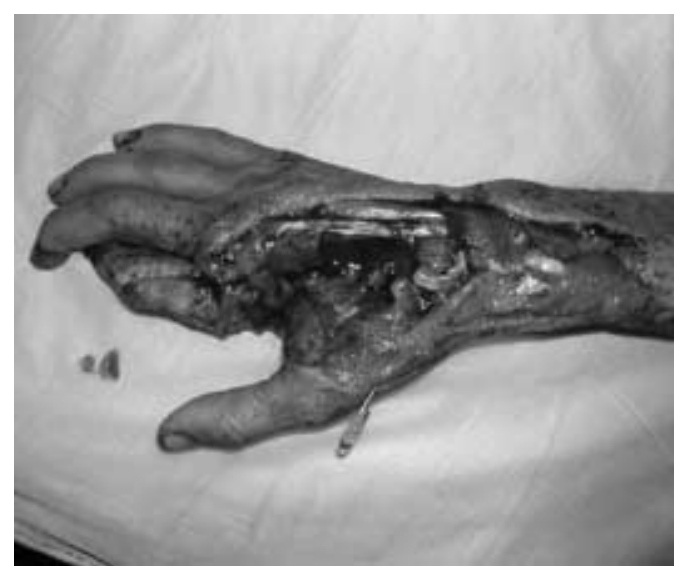

Fig 1.Shrapnel injury following debridement and $k$ wire stabilisation of thumb ray.

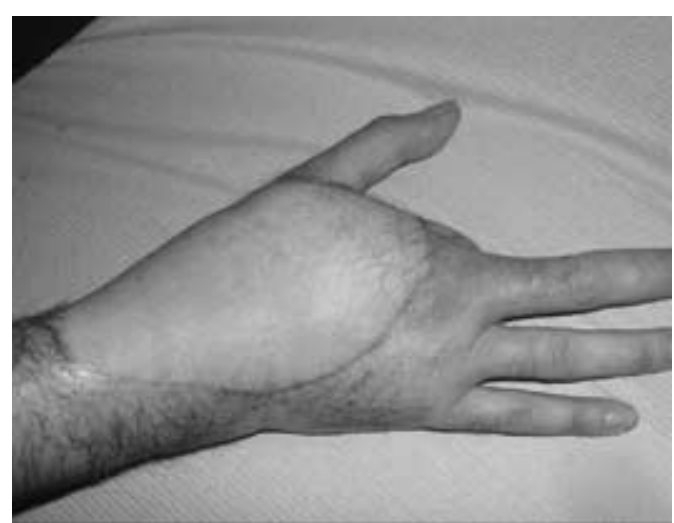

Fig 2. The reconstructed hand at 5 months after injury.

contaminated hand was attended to by debridement (Figure 1), amputation of the index finger, stabilisation of the thumb and third finger rays and coverage by a random pattern thoracoabdominal flap. This was performed in two stages with the thoracoabdominal flap being divided at 23 days and an abdominal advancement flap used to close the abdominal donor site. An excellent functional and cosmetic result was achieved (Figure 2).

\section{The Use Of Vacuum Assisted Closure For Open Abdominal Wounds}

\section{B Cooper, P Pillai, JP Garner}

\section{Introduction}

Vacuum-assisted closure (VAC) of open abdominal wounds has been developed to aid early fascial closure and prevent the need for further reconstructive surgery.

\section{Case Report}

A frail 72 year old lady underwent elective right hemi-colectomy via a transverse incision for a symptomatic ischaemic stricture. Post-operative complications led to two further laparotomies and extensive debridements. VAC therapy to her open 
abdomen was commenced on Day 17 and was eventually discontinued after a total of 25 days in situ. When reviewed six weeks later the wound had completely healed without the need for further intervention or reconstructive surgery.

\section{Discussion}

The commercial VAC system has been examined in 2 large studies: one of 55 patients with open abdomens following both trauma and elective surgery and another series of 71 patients. In both studies more than $80 \%$ of patients achieved fascial closure after VAC therapy. Complication and fistulation rates were low.

\section{Conclusion}

VAC therapy offers a successful, non-invasive method of managing open abdomens in patients not amenable to delayed primary closure. It may be a useful addition to the military surgeon's armamentarium.

\section{A Primary Fistula Associated With An Abdominal Aortic Aneurysm}

\section{GD Maytham}

A sixty-eight year old male with a previous history of a gastro-intestinal bleed presented with signs and symptoms of an abdominal aortic aneurysm. Investigations revealed the presence of an inflammatory process. CT imaging demonstrated a very large abdominal aortic aneurysm with features suggestive of an inflammatory cause, with blood in the stomach suggesting an aortoenteric fistula. Laparotomy revealed extensive inflammation and an aorto-enteric fistula, which was primarily repaired and oversewn from within the aneurysmal cavity. A tube graft was inserted. A relook laparotomy 48 hours later showed breakdown of the aorto-enteric fistula repair with blockage of the naso-jejunal catheter. A left axillo-bifemoral graft was inserted, followed by removal of the tube graft. A significant mass of clotted blood was present in the stomach, necessitating opening of the organ for evacuation. Further relook laparotomies performed over the next few weeks showed no further breakdown of the previous fistula site. The failure of the fistula repair in this patient may have occurred due to an increase in the duodenal intraluminal pressure secondary to the blockage of the naso-jejunal catheter and minimal drainage from the nasogastric catheter due to the presence of a large volume of coagulated blood in the stomach. This experience suggests that meticulous evacuation of any blood in the stomach, with gastrostomy placement at the time of the initial repair, may have avoided this complication.

\section{Traumatic Sub-Arachnoid Haemorrhage With Neurogenic Pulmonary Oedema: A Case Report}

\section{DM Taylor}

Both sub-arachnoid haemorrhage following trauma and pulmonary oedema following head injury are described in the literature in their own right. Each is moderately rare and carries a high mortality rate. Their presentation together is unusual and highly significant.

Our patient was presented to $A \& E$ by ambulance as a head injury following a witnessed low violence altercation. He was found to have GCS of 12 and was also acutely hypoxic despite supplementary oxygen. Initial chest $\mathrm{X}$-rays showed widespread pulmonary oedema and CT scanning revealed a "sub-arachnoid haemorrhage in the posterior fossa... with hydrocephalus"There was no skull fracture.

Cerebral angiogram showed an "irregular sacccular aneurysm arising from the posterior inferior cerebellar artery (PICA)". The aneurysm was catheterised and embolised with a coil in the aneurysm and parent vessel. Further CTs showed an extensive left sided PICA territory infarct with increased hydrocephalus. A right ventricular-peritoneal shunt was therefore inserted.

Following shunt insertion he made dramatic improvements with further slow but progressive recovery until fit for transfer to a rehabilitation unit 12 weeks after injury.

There is little mention of trauma as a cause of SAH in the standard texts, but it is recognised within the literature. However, doubts do exist as to whether this is 'cause or effect'.

Pulmonary oedema, along with myocardial complications, is felt to be secondary to massive catecholamine release, mediated by the anterior hypothalamus, leading to increased hydrostatic pressures and capillary permeability. These are reversible conditions with supportive treatment.

It is, therefore, useful to remember $\mathrm{SAH}$ following head injury and to suspect extracranial complications of intra-cranial injuries and to instigate aggressive, supportive treatment to avoid preventable death.

\section{Acute Lower Limb Compartment Syndrome After A Forced March}

\section{Weller}

A young army captain developed a case of Acute Limb Compartment Syndrome (ALCS) after a $13 \mathrm{~km}$ forced march. As it is 
very unusual to see ALCS in patients with no history of trauma, his diagnosis was delayed until 80 hours post injury, and consequently by the time he reached a Role 3 facility he had developed muscle necrosis and required four compartment decompression and excision of all the muscles in his anterior compartment.

The muscles in the leg are contained in a relatively inelastic fascia, and when an injury causes swelling of the muscles a vicious cycle starts which can eventually cause arteriolar compression and muscle necrosis. Patients present with severe pain which is out of proportion to the clinical situation and which continues to intensify after cessation of the activity. There is tenderness of the affected compartment, which is "glossy" and tense with hypoesthesia being a late sign. There may also be a low grade fever and raised white cell count. Pulses are usually present and if absent imply a vascular injury.

Although it is possible to measure compartment pressures the readings are often equivocal, and can lead to delayed diagnosis and treatment. ALCS can be diagnosed clinically and must be treated promptly with a fasciotomy, preferably four compartment via two full length incisions. Exertional ALCS is a rare event, but if the diagnosis is missed or delayed as in our case report, it can lead to a life-long disability which might otherwise be avoided.

\section{Role Of Gymnastics In The Army School Of Physical Training}

\section{DE Griffiths, R Hargrove, JC Clasper}

\section{Introduction}

As a result of a single serious neck injury seen at Frimley Park Hospital, we reviewed the injuries recorded at the Army School of Physical Training since December 1996.

\section{Methods}

This was a retrospective review of all acute accidents and injuries recorded in the Accident Book since its inception.

\section{Results}

Over $75 \%$ of the injuries that were serious enough to result in soldiers having their training terminated were as a direct result of gymnastic events such as vaulting, trampolining and somersaults. These events were also responsible for most of the small number of career threatening injuries.

\section{Discussion and Conclusions}

This raises questions about the inclusion of gymnastic events in course training programmes, especially when considering its relevance to army training in general.

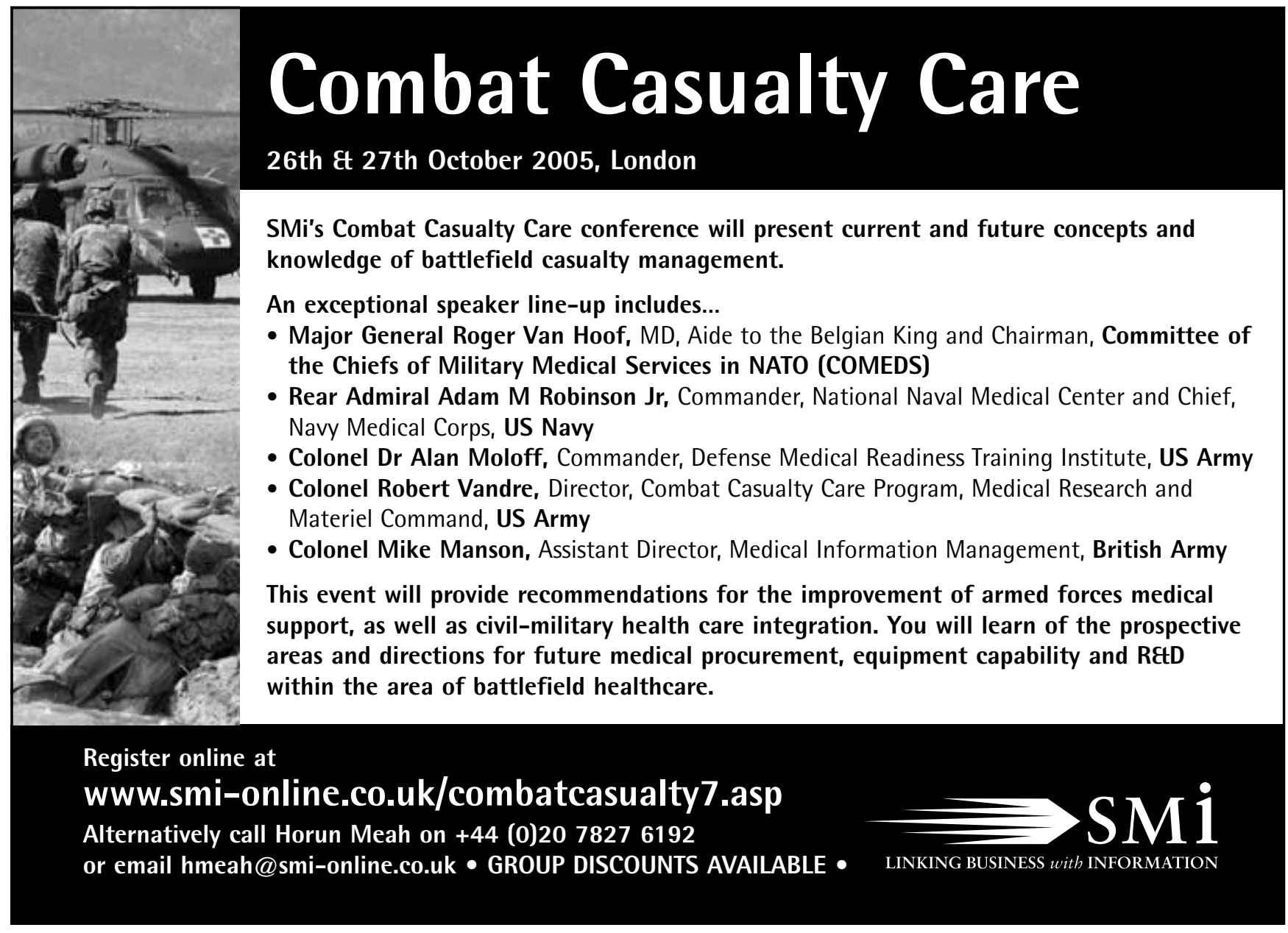

